\title{
Idiopathic Ventricular Fibrillation of the Brugada Type: An Atypical Form of Arrhythmogenic Right Ventricular Cardiomyopathy?
}

Brugada and Brugada (1) reported eight patients with idiopathic ventricular fibrillation (VF) who had characteristic electrocardiographic findings: right bundle bunchblock and persistent $\mathrm{ST}$ segment elevation in right precordial leads $\left(\mathrm{V}_{1}\right.$ to $\mathrm{V}_{2}-\mathrm{V}_{3}$ ). Although similar patients with idiopathic VF had been previously reported, Brugada and Brugada were the first to study it systematically. Since then, the Brugada type idiopathic VF has been the topic of major concern. In this issue of the Journal, Kobayashi et al (2) describe a patient with the Brugada type of idiopathic VF with an interesting familial history. Two members of the family died of heart disease and three members had an abnormal electrocardiogram: persistent ST segment elevation in the right precordial lead and right bundle branch block. Actually, familial occurrence and a family history of sudden death are clinical features of Brugada type idiopathic VT. A familial occurrence was noted in three of 8 patients in the report of Brugada and Brugada (1) and in $22 \%$ in our series.

See also p 637.

Brugada et al could not find any anatomical cardiac abnormality in their patients and thought that it was primarily an electrical disease. However, some believe that patients with Brugada type idiopathic VF have subtle underlying structural abnormalities of the right ventricle. In fact, three patients with Brugada type idiopathic VF reported by Martini et al (3) had subtle anatomical right ventricular abnormalities detected only after a detailed clinical investigation. We have also found slight right ventricular abnormalities in half of the patients with Brugada type idiopathic VF that were recognized only by detailed examinations by MRI and CT. Recently, Corrado et al (4) reported right ventricular dilatation and myocardial atrophy with adipose replacement of the right ventricular free wall as well as sclerotic interruption of the right bundle branch in a patient with familial Brugada type idiopathic VF. We also noted similar findings in two patients with Brugada type idiopathic VF: abnormal lipid accumulation only at the epicardial site of the right ventricle. Thus, it is now certain that half of the patients reported as Brugada type idiopathic VF are not idiopathic in the strict sense.
The clinical characteristics of the Brugada type idiopathic VF (right ventricular abnormalities with lipid accumulation, familial occurrence, male predominance) resembles those of arrhythmogenic right ventricular cardiomyopathy (5). But the difference between the two diseases is also noted. ST elevation in precordial leads is one of the characteristics in Brugada type idiopathic VF, while this characteristic is seldom found in patients with typical arrhythmogenic right ventricular cardiomyopathy. HV prolongation or RBBB is a common finding in patients with Brugada type idiopathic VF, as the case reported by Kobayashi et al in this journal, suggesting structural changes in the His-Pukinje system. In contrast, conduction abnormalities are usually confined to the right ventricular myocardium, manifested by delayed potential and fragmentation in patients with arrhythmogenic right ventricular dysplasia.

Thus, it is still premature to conclude that Brugada type idiopathic VF is one of the atypical types of arrhythmogenic right ventricular cardiomyopathy that has a polymorphic clinical spectrum, but it is an interesting hypothesis and worth investigation.

$$
\begin{array}{r}
\text { Tohru OHE, MD } \\
\text { Department of Cardiovascular Medicine, Okayama University } \\
2-5-1, \text { Shikadacho } \\
\text { Okayama } 700
\end{array}
$$

\section{References}

1) Brugada P, Brugada J. Right Bundle Branch Block, Persistent ST Segment Elevation and Sudden Cardiac Death: A Distinct Clinical and Electrocardiographic Syndrome. JACC 20: 1391, 1992.

2) Kobayashi $T$, Shintani U, Yamamoto $T$, et al. Familial occurrence of electrocardiographic abnormalities of Brugada type. Intern Med 35: 637, 1996.

3) Martini B, Nava A, Thiene G, et al. Ventricular fibrillation without apparent heart disease; Description of six cases. Am Heart J 118: 1203, 1989.

4) Corrado D, Nava A, Buja G, et al. Familial Cardiomyopathy Underlies Syndrome of Right Bundle Branch Block, ST Segment Elevation and Sudden Death. JACC 27: 443, 1996.

5) Marcus FI, Fontaine GH, Guiraudon G, et al. Right ventricular Dysplasia: A report of 24 adult cases. Circulation 65: 384, 1982. 